\title{
Psicologia e nutrição: predizendo a intenção comportamental de aderir a dietas de redução de peso entre obesos de baixa renda
}

\author{
Ana Paula Rodrigues Cavalcanti \\ Mardonio Rique Dias \\ Maria José de Carvalho Costa \\ Universidade Federal da Paraíba
}

\begin{abstract}
Resumo
O presente estudo foi realizado entre 189 obesos de baixa renda atendidos por nutricionistas na rede pública de saúde de João Pessoa, Paraíba. Levantou as crenças positivas e negativas e os referentes mais expressivos sobre estes pacientes quanto ao comportamento de seguir uma dieta médica para redução de peso. Um instrumento de medida da intenção comportamental de efetivamente seguir tais dietas foi construído, o que permitiu verificar a ênfase do aspecto normativo geral e atitudinal nos obesos com renda de até um saláriomínimo sobre sua intenção comportamental. Isto pode indicar dois pontos eficazes a serem explorados em comunicações dirigidas a esta classe social em campanhas de saúde para redução de peso. Esta medição tornase útil para ações preventivas de saúde; a obesidade é considerada uma epidemia mundial carente de controle imediato, aumentando em incidência principalmente nas classes mais baixas dos países em desenvolvimento.
\end{abstract}

Palavras-chave: obesidade; dieta; teoria da ação racional

\begin{abstract}
Psychology and nutrition: predicting behavioral intention to follow weight reduction diets among low-income obese patients. The present study was accomplished with 189 low income obese attended by public health nutritionists of João Pessoa, Paraíba. We found out positive and negative beliefs plus the most expressive referees on these patients about the behavior of following a medical diet to reduce weight. This permitted to build a behavioral intention measurement instrument to check those who might really follow these diets. It was verified the emphasis on general normative and attitudinal aspects over obese who had up to one minimum-wage income. This may indicate two efficacious points to be explored on targeted communications to this social class during weight loss public health campaigns. This measurement can be turned into preventive health actions; obesity is considered worldwide as epidemics, in need of immediate control, growing in incidence mainly among lower ranks of the population of developing countries.
\end{abstract}

Keywords: obesity; diet; theory of reasoned action

A Psicologia e a Nutrição são áreas do conhecimento separadas pela tradição da estrutura acadêmica, mas que inevitavelmente se unem no contexto da saúde pública, particularmente quando se observa o tratamento da obesidade (Sjöström, Yngve, Poortvliet, Warm, \& Ekelund, 1999).

Historicamente, a ciência médica diferenciou-se em várias especialidades baseadas na categorização de doenças, órgãos do corpo humano, idade, gênero e técnicas especiais. Os sistemas de saúde, órgãos de pesquisa e o próprio ensino médico ainda estão profundamente mergulhados nessa estrutura. Tal estrutura de organização causa a impressão de territorialismo, formando barreiras contra esforços conjuntos entre diferentes disciplinas. Entretanto, são as abordagens inter e multidisciplinares que podem superar os pontos críticos para o êxito nas pesquisas, na prevenção de doenças e promoção da saúde (Yngve et al., 1999).

Trabalhos recentes (Parra-Cabrera, Hernandez, DuranArenas, \& Lopez-Arellano, 1999; Pearce, 1996) sugerem que o estudo dos aspectos culturais e a incorporação de contribuições das ciências sociais terão importância cada vez maior no futuro para os trabalhos em problemas de saúde.

A população de baixa renda sofre de incidência de obesidade em proporções crescentes, fenômeno particularmente observado no Nordeste, até mesmo entre a população masculina. A Organização Mundial da Saúde (OMS) justifica este paradoxo do aumento da doença, tradicionalmente associada à fartura de alimentos, a mudanças na composição dietética 
da população ocidental e ao acesso barateado às farinhas e gorduras (OMS, 2001). O Ministério da Saúde do Brasil (2000) acresce a estes fatores a falta de orientação alimentar adequada e a redução da atividade física e do tamanho das famílias, que teriam aumentado a quantidade de alimentos disponíveis para as pessoas.

A predominância mundial da obesidade, seus malefícios decorrentes e os custos crescentes que as doenças correlatas trazem para os serviços de saúde tornaram-na equivalente ao tabagismo na sociedade atual, suplantando em projeções estatísticas preocupações sanitárias clássicas como a desnutrição e as doenças infecciosas e classificando-a atualmente como patologia prioritária para estudos científicos (OMS, 2001).

Cerca de 95\% dos obesos que seguem dieta para emagrecer fracassam na manutenção do corpo mais magro (OMS, 1997). Paralelamente, a incidência da obesidade aumenta mundialmente e nem a Medicina, nem a Nutrição, conseguem fazer esses pacientes mudarem suficientemente seus comportamentos alimentares (Le Barzic, 2001). Os programas de intervenção nutricional nem sempre alcançam sucesso. Dentre outros motivos, estaria a compreensão insuficiente da relação entre os comportamentos ligados à saúde e o tratamento prescrito. Apesar desse reconhecimento, os causadores potenciais da patologia são enfrentados quase exclusivamente com prescrições científicas de dietas (Crawford, Jeffery, \& French, 2000).

Deduz-se desta exposição que o tratamento da obesidade, na forma como é tradicionalmente conduzido pela Medicina e pela Nutrição, certamente conseguirá resultados inexpressivos. Isso parece ser confirmado pelo reconhecimento das altas taxas de reincidência da obesidade e pelos estudos mais recentes conduzidos pela própria OMS investigando os aspectos sociológicos, econômicos, culturais e psicológicos da doença, almejando através da multidisciplinariedade obter uma melhora nos indicadores epidemiológicos.

Especificamente quanto à mudança comportamental para promoção de hábitos saudáveis e aderência terapêutica, quatro quadros teóricos estudados em amplitude e profundidade em Psicologia Social são considerados como os principais: o modelo de crenças em saúde, de Rosenstock (1966), a teoria cognitiva social, de Bandura (1986), a teoria da ação racional, de Fishbein e Ajzen (1975) e a teoria da ação planejada, de Ajzen e Madden (1986). As extensas pesquisas baseadas nestas teorias indicam que as crenças em saúde influenciam a decisão de assumir comportamentos preventivos. A intenção comportamental seria uma variável interveniente importante no estudo dos comportamentos em saúde, e o encorajamento de processos auto-regulatórios da conduta poderia traduzirse em ações preventivas. A utilidade das medições de um dado comportamento tem sido demonstrada em uma série de comportamentos ligados à saúde (Fisher \& Fisher, 2001).

As diferenças básicas entre os modelos são, dentre outras, que o modelo de crenças em saúde permite auxílio na promoção da aderência terapêutica e na tomada de decisões em políticas públicas de saúde. Foi o primeiro modelo de mudança comportamental em saúde e continua aceito integralmente por vários pesquisadores da área até os dias atuais, mostrando-se especialmente adequado para a criação de políticas públicas em saúde, por não restringir ou recomendar propostas específicas de intervenção.

A teoria cognitiva social, de Bandura (1986), adequa-se muito bem à verificação de comportamentos que não dependem exclusivamente da vontade do indivíduo. Porque utiliza o conceito de auto-eficácia percebida para exercer um determinado comportamento, oferece uma visão mais individualista para a modificação da conduta. Também não especifica uma estratégia de intervenção para conduzir uma modificação comportamental.

A teoria da ação racional (TAR) é específica para condutas sob controle da vontade do indivíduo, e seu quadro teórico estabelece construtos a serem medidos, ponderados e utilizados em comunicações persuasivas que levem à mudança comportamental.

Por fim, a teoria da ação planejada (TAP) busca suplementar a TAR, da qual deriva, através da possibilidade de modificação também daqueles comportamentos que fogem ao controle pela vontade pessoal (Fisher \& Fisher, 2001). Busca suplementá-la na capacidade de predição comportamental através da inclusão do conceito de controle comportamental percebido.

Diante da gravidade das considerações sobre a obesidade expostas pela Medicina, da insuficiência das recomendações médico-nutricionais para alcançar a melhoria duradoura do estado de saúde dos obesos, da necessidade que a Nutrição tem de contribuições da Psicologia para explicar e modificar hábitos prejudiciais à redução do peso entre estes pacientes e da capacidade da Psicologia Social de estruturar uma intervenção concernente que permita comparações de variância, a TAR e a TAP surgem como quadros teóricos elegíveis para este intento.

A TAP já foi empregada em estudos anteriores (Povey, Conner, Sparks, James, \& Shepherd, 2000; Schifter \& Ajzen, 1985), que investigaram a redução de peso e seu pressuposto de que a aderência terapêutica a dietas de redução de peso deve ser categorizada como não sujeita ao controle pela vontade pessoal permanece válido.

A TAR foi testada quanto à redução de peso entre universitárias norte-americanas (Sejwacz, Ajzen, \& Fishbein, 1980), e os resultados corroboraram a premissa do modelo de que a medida da atitude e da norma subjetiva permitem predizer com exatidão a intenção comportamental; entretanto o estudo reconheceu que um programa de emagrecimento é constituído de uma série de comportamentos que produzem perda de peso (por exemplo, exercício físico e mudança de hábitos alimentares) que necessitam de mensuração separada específica.

O presente estudo objetivou esclarecer quais construtos do modelo da TAR apresentam maior peso na equação mensuradora da intenção comportamental de seguir uma dieta para emagrecimento entre obesos de baixa renda atendi- 
dos nos serviços de saúde pública, obtendo, ao final desta equação, uma previsão do grau da intenção comportamental para aderência terapêutica. Esta informação permitiria a estruturação de comunicações persuasivas numa futura intervenção, propiciando uma mudança de conduta que levasse a uma maior persistência no tratamento da obesidade.

Para isso, os objetivos específicos foram: identificar os referentes (pessoas mais importantes) modais salientes (mais expressivos), as crenças positivas e negativas quanto ao comportamento de seguir uma dieta para emagrecimento e, a partir dessa identificação, segundo as orientações da teoria, construir e validar um instrumento de medida da intenção comportamental específica, seguido das ponderações da atitude e da norma subjetiva.

A primeira etapa consistiu no levantamento das crenças e dos referentes modais salientes; a segunda, na construção e aplicação de um questionário sobre a perda de peso através de dieta, medindo a força das crenças mais freqüentes e a magnitude da disposição para concordar com a norma subjetiva preponderante (a opinião dos referentes modais salientes).

\section{Método}

\section{Amostra da etapa 1}

Na primeira etapa utilizou-se uma amostra aleatória de 41 pacientes obesos atendidos nos ambulatórios de Nutrição do Hospital Universitário Lauro Wanderley $(n=21)$ e no Centro de Atendimento Médico Especializado (CAME) de Jaguaribe $(n=21)$, em João Pessoa, Paraíba. Os participantes tinham a idade mínima de 18 anos, e foram excluídos os diabéticos, cardiopatas, gestantes, portadores de disfunções endócrinas ou em uso de medicação interferente no apetite ou no metabolismo nutricional, para afastar a hipótese de que suas crenças a respeito de perder peso seguindo dietas estivessem influenciadas pelo efeito coadjuvante dos medicamentos ou pelos efeitos deletérios destas patologias. Houve predominância do sexo feminino, da faixa etária de 31 a 60 anos, do nível escolar fundamental (completo ou não) e de renda média de 3 salários-mínimos. Estas características são corroboradas pela comparação com os resultados da Pesquisa Nacional por Amostra de Domicílios, de 1998, do IBGE.

\section{Instrumento 1: a entrevista}

Os pacientes responderam a uma entrevista aberta, que continha levantamento de dados sócio-demográficos e três questões sobre o tema investigado ("Na sua opinião, quais as vantagens de diminuir o peso pela dieta? Na sua opinião, quais as desvantagens de diminuir o peso pela dieta? Que pessoas são importantes para você, quando dão uma opinião sobre este assunto?”). As respostas a cada uma das três perguntas foram tabuladas pela freqüência, compondo, conseqüentemente, três listas que foram submetidas à categorização por três psicólogos sociais não pertencentes ao grupo de trabalho, que atuaram como juízes. Cada juiz definiu quais as dimensões que a seu ver estariam se salien- tando nestas listas, e elaborou um resumo com a respectiva contagem de freqüência por categoria.

Reunidas estas categorizações individuais, buscaram-se os pontos de convergência entre os resumos, definindo uma segunda categorização, final, que serviu para elaborar o instrumento de medida das variáveis preditoras da teoria (atitude, norma subjetiva, crenças comportamentais, crenças normativas e intenção de adotar o comportamento-alvo).

No presente estudo, as classificações de cada juiz convergiram sobre: (a) quanto aos referentes - a categorização deveria ser feita quanto à freqüência de contato ou grau de parentesco; (b) quanto às crenças negativas - a categorização deveria ser feita quanto às dimensões de privação/saciedade; controle; efeitos físicos; mudanças na dimensão social e psicológica da alimentação; (c) quanto às crenças positivas - a categorização deveria ser feita quanto às dimensões de efeitos sobre a saúde; melhor aparência física; efeitos físicos e psicológicos sobre o respondente; criação de hábitos considerados saudáveis.

\section{Instrumento 2: o questionário}

A partir das convergências das classificações dos juízes, construiu-se um questionário final de 30 itens, com escala tipo Likert de cinco pontos (nos estudos anteriores que empregaram a TAR entre indivíduos com pouca educação formal, a colocação de cinco intervalos foi recomendada), na qual o extremo de algarismo 1 representa a maior força, e seu oposto, o algarismo 5 , a menor. O algarismo 3 , central, uma situação neutra ou indiferente por parte do respondente. Os algarismos 2 e 4, respectivamente, indicam uma tendência à maior ou menor força. Na etapa do tratamento estatístico dos dados, as escalas foram revertidas para realçar o aspecto de que quanto maior as médias, mais positivo o julgamento dos respondentes.

A medida do construto atitude está representada pelos itens 1 a 4, a força das crenças comportamentais positivas e negativas pelos itens 5 a 10, a avaliação das conseqüências das crenças comportamentais pelos itens 11 a 16, a norma subjetiva por um único item, o 17 , os referentes estão representados pelas crenças normativas (itens 18 a 23), a motivação para concordar com os referentes, pelos itens 24 a 29, e a intenção comportamental por um único item, o 30.

A força das crenças foi avaliada através de extremos denominados possível e impossível, substituindo os termos mais comumente utilizados - provável e improvável diante da previsão de um baixo nível de escolaridade e renda da amostra.

A atitude, conforme a teoria, é medida em quatro dimensões pré-definidas, representadas semanticamente pelos enunciados dos próprios respondentes da etapa 1 (útil/inútil; bom/ruim; agradável/desagradável; aconselhável/desaconselhável).

A cada item do construto das crenças comportamentais correspondeu um item semelhante para avaliação das conseqüências.

A forma de apresentação dos itens no questionário é ilustrada na Figura 1. 
1. Eu fazer regime para emagrecer nos próximos meses é:

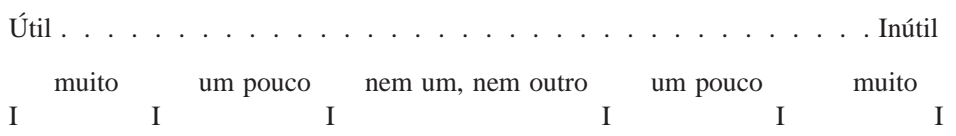

6. Para emagrecer, eu vou ter que controlar o que eu como:

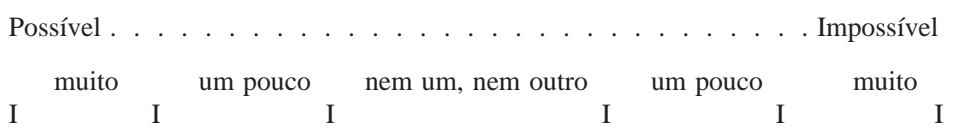

12. Ter que controlar minha comida é:

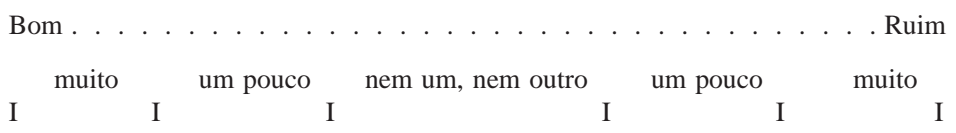

Figura 1. Exemplos da forma de apresentação dos itens no questionário.

A cada crença normativa correspondeu um item medindo a motivação para concordar com aquele referente:

19. Meus pais acham que eu devo fazer regime para emagrecer: (Possível... Impossível, etc.)

25. Quase sempre eu concordo com o que meus pais acham que eu devo fazer:

(Possível... Impossível, etc.)

A norma subjetiva e a intenção comportamental são itens de medição única:

17. A maioria das pessoas que são importantes para mim acha que eu devo fazer dieta para emagrecer:

(Possível... Impossível, etc.)

30. Eu pretendo fazer regime para ficar mais magro nos próximos meses:

(Possível... Impossível, etc.)

\section{Amostra da etapa 2}

Entrevistou-se um total de 189 pacientes obesos, com os mesmos critérios de inclusão e exclusão da primeira etapa, atendidos nos ambulatórios de nutrição do CAME de Jaguaribe ( $n=73)$, do CAME Primavera $(n=98)$, na Unidade de Saúde Lourival Gouveia Moura, do bairro de Mandacaru ( $n=13)$ e no Hospital Universitário Lauro Wanderley $(n=5)$, em João Pessoa, Paraíba.

Verificou-se mais uma vez a predominância do sexo feminino (98,9\% da amostra), idade média de 37 anos ( $D P=$ 12,43), nível de instrução majoritário médio, completo ou não (38,6\%), e renda familiar média de 1 a 2 salários-mínimos (34,4\%). A Pesquisa Nacional por Amostra de Domicílio PNAD do IBGE (1998) ratifica esta qualificação, sustentando, entretanto, que não existe predominância de nenhum dos sexos no uso do serviço público de saúde do país. Vinte e oito sujeitos foram excluídos da amostragem por analfabetismo (perfariam 15,1\% da amostra utilizada). Na primeira etapa, nenhum respondente analfabeto foi encontrado, o que poderia ser justificado pela amplitude da amostra final ou ainda pelo alto índice de analfabetismo da população paraibana e a presença de usuários do interior do Estado, onde a incidência de analfabetismo é maior.

O tempo médio de preenchimento foi de 9 minutos. Em um dos postos de saúde havia uma sala reservada para os trabalhos, para onde eram encaminhados os pacientes logo após a consulta com a nutricionista. Em outro, os sujeitos preencheram seus questionários em uma cadeira, num canto da própria sala do nutricionista. O terceiro teve seus questionários aplicados na fila de espera de atendimento, em frente à sala do médico, de portas abertas.

Fishbein e Ajzen (1975) recomendam que se verifiquem primeiro as relações existentes entre todas as variáveis independentes (atitude, norma subjetiva, crenças comportamentais versus avaliação das conseqüências destas crenças e crenças normativas versus motivação para concordar com elas) que compõem o modelo teórico e a variável dependente (a intenção comportamental), verificando a força e o tipo dessas relações. Para isto, utilizou-se o coeficiente de correlação $r$, de Pearson, com seus respectivos níveis de significância $p$, calculado através do software SPSS, versão 7.5. Após a verificação da força e do tipo de relação existente entre as variáveis, recomenda a teoria que se busque a predição da variável-critério (a intenção comportamental), através da ponderação das mesmas variáveis independentes. Para isso utilizou-se regressão múltipla, método stepwise.

\section{Resultados}

Verificou-se que tanto a atitude quanto o aspecto normativo social influenciam a intenção comportamental em estudo. A crença normativa geral respondeu por $17 \%$ da variância e a atitude, $6 \%$, totalizando $23 \%$ de poder de predição. 
A Tabela 1 mostra que existe uma correlação positiva forte entre os construtos atitude e crença normativa geral, o que não é comum nos estudos que utilizam a TAR. Geralmente essa correlação se dá entre a atitude e a crença comportamental geral, de acordo com o que estabelece a teoria.

A atitude e a intenção, a intenção e a crença normativa geral, e por fim a norma subjetiva e a crença normativa geral correlacionaram-se significativamente (acima de 0,30). Estas correlações, entretanto, já eram esperadas dentro do modelo teórico. Os estudos já existentes empregando a TAR comumente mostram a norma subjetiva correlacionando-se de forma substancial com a intenção, mas tal não se deu neste trabalho.
Quando foram isoladas as faixas de renda consideradas baixas, consoante os objetivos deste estudo, verificou-se que o segmento de renda familiar de até um salário mínimo apresentou as correlações mais altas, conforme se pode atestar na Tabela 2.

Novamente, os construtos atitude e intenção, e crença normativa geral e intenção mostraram-se positiva e significativamente correlacionados. Esperava-se que a norma subjetiva apresentasse valores relevantes, o que não ocorreu.

A Tabela 3 exclui as variáveis norma subjetiva e crença comportamental geral, concluindo que apenas as variáveis preditoras crença normativa geral e atitude entram na equação de regressão, explicando um total de 23,1\% da variância

Tabela 1

Correlações entre a variável dependente (intenção comportamental de fazer dieta para reduzir o próprio peso) e as variáveis independentes: crença comportamental geral, crença normativa geral, atitude e norma subjetiva; na amostra total $(N=189)$

\begin{tabular}{lcccc}
\hline & INTENÇÃO & CRCOMGE & CRNORGE & ATITUDE \\
\hline INTENÇÃO & - & & & \\
CRCOMGE & 0,118 & - & & \\
CRNORGE & $0,413^{*}$ & $0,269^{* *}$ & - & \\
ATITUDE & $0,340^{* *}$ & $0,203^{* *}$ & $0,374^{* *}$ & - \\
NORMSUB & $0,168^{*}$ & 0,134 & $0,420^{* *}$ & $0,226^{*}$ \\
\hline
\end{tabular}

Nota. CRCOMGE $=$ crenças comportamentais gerais; CRNORGE $=$ crenças normativas gerais; NORMSUB $=$ norma subjetiva.

$* p<0,05 \quad * * p<0,01$

Tabela 2

Correlações entre a variável dependente "intenção comportamental de fazer dieta para reduzir o próprio peso" e as variáveis independentes: crença comportamental geral, crença normativa geral, atitude, e norma subjetiva; na faixa de renda baixa (até um saláriomínimo, $n=46$ )

\begin{tabular}{lcccc}
\hline & INTENÇÃO & CRCOMGE & CRNORGE & ATITUDE \\
\hline INTENÇÃO & - & & & \\
CRCOMGE & 0,048 & - & & \\
CRNORGE & $0,335^{*}$ & 0,117 & - & \\
ATITUDE & $0,559 * *$ & 0,022 & 0,078 & - \\
NORMSUB & 0,149 & 0,174 & 0,166 & 0,158 \\
\hline
\end{tabular}

Nota. CRCOMGE = crenças comportamentais gerais; CRNORGE $=$ crenças normativas gerais; NORMSUB $=$ norma subjetiva.

$* p<0,05 \quad * * p<0,01$ 
compartilhada da intenção comportamental de fazer dieta para reduzir o próprio peso. Houve preponderância do construto crença normativa geral, responsável por $17 \%$ desse valor, apresentando-os, então, como os construtos de maior influência sobre a intenção comportamental.

Os resultados obtidos com a análise das correlações e regressões múltiplas mostram que, de acordo com Tabachnick e Fidell (1996), o tamanho da amostra está suficiente, visto que aconselham uma razão igual a $N>50+8 m$ de sujeitos, onde $m$ é igual ao número de VIs, ou seja, para o presente estudo, uma razão igual a $N>50+8$ x 4, ou $N>82$ sujeitos. Neste trabalho já se encontra um $N=189$.

Esperava-se, segundo as premissas da TAR, que a norma subjetiva e a atitude aparecessem juntas na equação de regressão, mas tal não se deu.

Quando se consideram apenas as faixas de renda consideradas baixas, de acordo com os objetivos deste trabalho, novamente percebe-se que a correspondente aos ganhos de até um salário-mínimo apresenta os resultados mais expressivos (Tabela 4).

A variância explicada pelas variáveis atitude e crença normativa geral aumentou para 38,7\% nesta faixa da amostra, onde cerca de 30\% é devida à variável atitude, e 8,7\% creditados à variável crença normativa geral, numa hierarquia inversa à da Tabela 3.

\section{Discussão}

Observou-se que os obesos que utilizam os serviços públicos de saúde de João Pessoa, nesta amostragem, não percebem pressão relevante do seu meio social de uma forma generalizada (o que seria detectado pela norma subjetiva). O estudo de Ross (1994) confirma que à medida que aumenta a renda e o nível de instrução pessoal do obeso, mais cresce a pressão percebida ao seu redor, com limites menos definidos, para que emagreça. Wadden e Stunkard (1987) referem que os estudantes universitários, por exemplo, apresentam uma alta lipofobia. Field et al. (1999) correlacionam o desejo e a pressão para emagrecer com o gênero e a idade: quanto mais jovem as respondentes mulheres, maior a vontade de perder peso e maiores os níveis de depressão, ansiedade e angústia por não corresponder aos ideais estéticos divulgados pela mídia.

$\mathrm{O}$ construto atitude, que aqui surge com destaque na predição do comportamento-alvo, igualmente salientouse nos trabalhos de Schifter e Ajzen (1985) e Povey et al. (2000), utilizando a teoria da ação planejada. Nesses estudos, porém, a norma subjetiva ocupou o lugar da crença normativa geral aqui destacada. Isto poderia dever-se

Tabela 3

Regressões múltiplas para os construtos do comportamento de "fazer dieta para reduzir o próprio peso" na amostra total $(N=189)$

\begin{tabular}{cccccccc}
\hline VI & $R$ & $R^{2}$ & $F$ & Sig $(F)$ & Beta & T & $p$ \\
\hline CRNORGE & $0,413^{\mathrm{a}}$ & 0,171 & 31,329 & $0,000^{\mathrm{a}}$ & 0,413 & 5,597 & 0,000 \\
ATITUDE & $0,480^{\mathrm{b}}$ & 0,231 & 22,616 & $0,000^{\mathrm{b}}$ & 0,263 & 3,420 & 0,000 \\
\hline
\end{tabular}

Nota. Variável dependente = intenção; VI = variável independente; CRNORGE = crença normativa geral.

${ }^{a}$ Crença Normativa Geral

${ }^{\mathrm{b}}$ Crença Normativa Geral + Atitude

Tabela 4

Regressões múltiplas para os construtos do comportamento de "fazer dieta para reduzir o próprio peso” na faixa de renda baixa (até um salário-mínimo, $n=46$ )

\begin{tabular}{cccccccc}
\hline V I & $R$ & $R^{2}$ & $F$ & Sig $(F)$ & Beta & T & $p$ \\
\hline ATITUDE & $0,547^{\mathrm{a}}$ & 0,299 & 16,236 & $0,000^{\mathrm{a}}$ & 0,547 & 4,060 & 0,000 \\
CRNORGE & $0,622^{\mathrm{b}}$ & 0,387 & 11,696 & $0,000^{\mathrm{b}}$ & 0,298 & 2,305 & 0,000 \\
\hline
\end{tabular}

Nota. Variável dependente = intenção; VI = variável independente.

a Atitude

${ }^{\text {b }}$ Atitude + Crenças Normativas Gerais 
à faixa de renda sob estudo (a média foi de dois saláriosmínimos, chegando a abranger em torno de 70\% da amostra, quando o valor se estendeu até quatro salários-mínimos), ou ainda apresentar-se como sinal de desejabilidade social aumentada pelas condições ambientais durante a aplicação do instrumento de medida . Ou seja, o respondente atribuiria ao médico o papel de representante dos seus referentes pessoais mais expressivos, e que estaria naquele momento da consulta (e do preenchimento do questionário) reiterando com outros métodos e informações o que essas pessoas do seu convívio pessoal esperavam que ele fizesse ou pensasse; mas não perceberia no nutricionista uma tradução do que a sociedade lhe oferecia em sentido amplo para solução do seu problema de saúde.

Igualmente, pode-se diante destes dados questionar se o construto controle comportamental percebido, da teoria da ação planejada, teria de fato relevância na predição do comportamento de seguir dietas de emagrecimento, conforme defendido por Schifter e Ajzen (1985) e Povey et al. (2000) em seus estudos correlatos. A Atitude e a pressão social percebida (individualizada ou coletiva) salientaram-se com o uso de ambas teorias, porém os resultados ainda não permitem concluir a substancialidade do argumento de que fazer dieta seria um comportamento para além da vontade individual de assumi-lo.

Estes resultados atestam que a teoria da ação racional, para este comportamento específico e neste estudo, ainda único no Brasil, explica a intenção de efetivamente seguir uma dieta de perda de peso receitada por nutricionista num limiar mais baixo do que o exibido nos estudos feitos com outros comportamentos ligados à saúde. A TAR costuma apresentar resultados maiores que $30 \%$ de variância explicada, e a isto também se deve sua longevidade.

Tal diminuição no poder de predição neste estudo pode dever-se aos seguintes fatores:

(a) Faixa de renda da amostra. Todas as faixas de renda estiveram representadas entre os respondentes, assim como todos os níveis educacionais. Esta abrangência foge ao escopo definido para estudo e dá margem a diversas interpretações dos resultados estatísticos, ao se considerarem as influências e particularidades de cada estrato social, econômico e cultural considerado.

(b) Tipo de escala usada no instrumento. Uma escala de sete, em vez de cinco pontos, talvez permitisse maior discriminação nas respostas. Porque foi suposto no delineamento inicial do estudo que a baixa renda dos usuários de postos de saúde deveria corresponder a uma escolaridade igualmente reduzida, preferiu-se encurtar a escala para evitar dificuldades de preenchimento entre os respondentes. Contrariamente ao esperado, verificouse que o nível de instrução da maioria não era precário; era de $2^{\underline{0}}$ grau completo, ou incompleto, em 38,6\% dos casos. Normalmente, os estudos da TAR utilizam uma escala tipo Likert de sete pontos. (c) Tipo de comportamento estudado. A obesidade é uma doença eivada de particularidades sociais, culturais e históricas e o modelo é considerado parcimonioso em construtos. Apesar de esta característica facilitar as comparações entre os resultados de vários trabalhos, poderia não contemplar de maneira suficiente, ou adequada, outros aspectos relevantes do comportamento estudado.

(d) Local do levantamento dos dados. A maioria dos instrumentos foi aplicada logo após a consulta, sob as vistas do médico (116 dos respondentes, ou 62\% da amostra), o que pode ter aumentado a desejabilidade social e, conseqüentemente, a homogeneidade das respostas. O serviço público de saúde muito raramente dispõe de instalações mais bem adequadas para a pesquisa científica. A maioria destes casos foi registrada no CAME Primavera, onde o próprio nutricionista incitava o paciente a aderir estritamente à dieta imediatamente antes de indicar o local onde estava o pesquisador com o questionário. Conforme o estudo de Wadden e Stunkard (1993), a atitude do profissional de saúde tem influência sobre a auto-percepção do obeso e sobre a interpretação do tratamento que lhe está sendo dispensado.

(e) Redação do item de intenção comportamental. $\mathrm{O}$ item correspondente à intenção comportamental (item 30), apesar de corresponder ao alvo, ação, contexto e tempo recomendados na teoria ("Eu pretendo fazer regime para ficar mais magro nos próximos meses”), poderia ser modificado em sua redação para provocar um estímulo maior e mais específico. Por exemplo: "Eu pretendo fazer regime para emagrecer, do jeito que a doutora me disser que é pra fazer, nos próximos meses”. De acordo com o estudo correlato de Sejwacz et al. (1980), o conceito de fazer dieta pode ter significados diferentes para pessoas diferentes. Em seu trabalho, esses autores utilizaram além de um item específico para medir a intenção comportamental de fazer dieta, outros itens complementares perguntando sobre a intenção de adotar comportamentos específicos necessários para manter uma dieta (por exemplo, evitar beliscar entre as refeições) e encontraram uma correlação alta $(r=0,60)$ entre esses índices.

$\mathrm{O}$ estudo mencionado, entretanto, foi realizado entre universitárias de Massachusetts e utilizou uma amostra de voluntárias que se consideravam obesas, não tinham orientação médica ou nutricional para redução de peso, e tencionavam emagrecer nos dois meses seguintes ao do preenchimento do questionário fazendo exercícios físicos e dieta por conta própria. As correlações verificadas entre a atitude, a norma subjetiva, a intenção e o peso efetivamente perdido foram baixas (as maiores reduções de peso foram de meio quilo, com apenas $8 \%$ de explicação da variância).

$\mathrm{Na}$ amostra de interesse específico deste estudo (obesos de baixa renda), a teoria mostrou-se especialmente satisfatória na sua capacidade de predição da intenção comportamental na faixa de renda de até um salário-míni- 
mo (12,7\% da amostragem): 39\% de explicação da variância, com maior peso para os construtos da atitude e da crença normativa geral. Esta parte da população representa um terço (33\% das mulheres e 31\% dos homens) da população economicamente ativa na Paraíba.

Tal diferença no desempenho da teoria pode dever-se às especificidades deste segmento social: composto por pessoas de nível educacional baixo, a escala de cinco pontos teria servido então com mais eficácia. É útil lembrar que a desejabilidade social diminui na classe baixa e aumenta na classe média, declinando novamente nos estratos de renda mais altos, e que isto teria afetado positivamente os resultados.

\section{Conclusão}

Estes novos dados sobre o comportamento dos obesos muito pobres que recebem dieta médica para emagrecer já indicam dois enfoques para uma intervenção em saúde pública. Em primeiro lugar, a atitude destes obesos para com seu próprio regime de emagrecimento é crucial para o sucesso do tratamento da obesidade. Atitudes negativas a este respeito poderiam diminuir em freqüência se mais informações sobre as vantagens de perder peso a curto e longo prazo chegassem ao seu conhecimento através de programas de saúde que incluíssem trabalhos de orientação psicológica e educativa para esta população.

Em segundo lugar, a opinião alheia das pessoas que fazem parte do convívio diário desses pacientes sobre sua necessidade de emagrecerem tem forte influência sobre eles. Esta constatação, além de útil para elaborar programas de intervenção, pode ser de relevância para os atendimentos individualizados dos psicólogos da rede pública de saúde.

A validade externa destas conclusões, entretanto, estaria na dependência de pesquisas posteriores similares, já que o poder de generalização da TAR restringe-se à amostra estudada, conforme advertem seus autores.

As atuais campanhas contra hipertensão e diabetes tipo 2, que seguem orientações gerais nacionais em que se recomenda o controle imediato da obesidade, todavia sem estratégias específicas de ação, igualmente se beneficiaram destas novas informações, podendo reduzir os custos e o tempo do tratamento dessas patologias.

Resta, por fim, elucidar qual a melhor forma de persuadir esses pacientes sobre as vantagens de aderir à dieta médica: se através de comunicações persuasivas positivas ou negativas; o que já enseja a continuação do presente estudo, executando a última etapa estabelecida pela TAR.

\section{Agradecimentos}

A realização desta pesquisa foi possível graças ao suporte mantido pela CAPES, órgão governamental ao qual esta equipe de pesquisa deve o apoio e o incentivo ao trabalho científico.

\section{Referências}

Ajzen, I., \& Madden, T. (1986). Prediction of goal-directed behavior: attitudes, intentions, and perceived behavioral control. Journal of Experimental Social Psychology, 22, 453-474.

Bandura, A. (1986). Social foundations of thought and action. Englewood Cliffs, New Jersey: Prentice Hall.

Crawford, D., Jeffery, R. W., \& French, S. A. (2000). Can anyone successfully control their weight? Findings of a three year communitybased study of men and women. International Journal of Obesity, 24(9), 1107-1110.

Field, A. E., Cheung, L., Wolf, A. M., Herzong, D. B., Gortmaker, S. L., \& Colditz, G. A. (1999). Exposure to the mass media and weight concerns among girls. Pediatrics, 103(3), 36-39.

Fishbein, M., \& Ajzen, I. (1975). Belief, attitude, intention and behavior: an introduction to theory and research. Reading, Massachusetts: Addison-Wesley.

Fisher, D. J., \& Fisher, W. A. (2001). Theoretical approaches to individual-level change in HIV-risk behavior. In J. Peterson \& R. DiClement (Orgs.), HIV prevention handbook (pp. 89-115). Nova York: Plenum.

Instituto Brasileiro de Geografia e Estatística - IBGE (1998). Pesquisa nacional por amostra de domicílios. 4 - trabalho. Retirado em 11.junho.2001 do website http://www.ibge.gov.br/ibge/estatistica/populacao/ trabalhoerendimento/penad.

Le Barzic, M. (2001). The syndrome of cognitive restraint: from the nutritional standard to eating disorders. Diabetes Metabolism, 27(4), 512-516.

Ministério da Saúde (2000). Obesidade e desnutrição. NUT/FS/UnB-ATAN/ DAB/SPS. Retirado em 02.setembro.2002 do website http:// www.saude.gov.br/sps/areastecnicas/carencias/documentos/ obesidade_desnutricao.pdf.

Organização Mundial da Saúde - OMS (1997). Obesity: Preventing and managing a global epidemic. Report of a WHO consultation on obesity. Retirado em 28.setembro.2001 do website http://www.who/nut/ncd/98.1.

Organização Mundial da Saúde - OMS (2001). Obesity: Controlling the global epidemic. Retirado em 28.setembro.2001 do website http://www.who.int/ nut/obs.htm.

Parra-Cabrera, S., Hernández, B., Durán-Arenas, L., \& López-Arellano, O. (1999). Modelos alternativos para el análisis epidemiológico de la obesidad como problema de salud pública. Revista de Saúde Pública, 33(3), 314-325.

Pearce, N. (1996). Traditional epidemilogy, modern epidemiology, and public health. American Journal of Public Health, 86, 678-683.

Povey, R, Conner, M., Sparks, P., James, R., \& Shepherd, R. (2000). Application of the theory of planned behavior to two dietary behaviors: roles of perceived control and self-efficacy. British Journal of Health Psychology, 5(2), 121-139.

Rosenstock, I. M. (1966). The health belief model: explaining health behavior through expectancies. In K. Glanz, F. M. Lewis, \& B. K. Rimer (Orgs.), Health behavior and health educations: theory, research and practice (pp. 39-62). São Francisco: Jossey-Bass.

Ross, C. E. (1994). Overweight and depression. Journal of Health and Social Behavior, 35(1), 63-79.

Schifter, D. B., \& Ajzen, I. (1985). Intention, perceived control, and weight loss. An application of the theory of planned behavior. Journal of Personality and Social Psychology, 49, 843-851.

Sejwacz, D., Ajzen, I., \& Fishbein, M. (1980). Predicting and understanding weight loss: intention, behaviors and outcomes. In I. Ajzen \& M. Fishbein (Orgs.), Understanding attitudes and predicting social behavior (pp. 111130). Englewood Cliffs, New Jersey: Prentice Hall. 
Sjöström, M., Yngve, A., Poortvliet, E., Warm, D., \& Ekelund, U. (1999). Diet and physical activity - interactions for health; public health nutrition in the European perspective. Public Health Nutrition, 2, 453-459.

Tabachnik, B. G., \& Fidell, L. S. (1996). Using multivariate statistics. Nova York: Harper Collins.

Wadden, T. A., \& Stunkard, A. J. (1987). Psychopathology and obesity. Annual NewYork Academical Sciences, 499, 55-65.
Wadden, T. A., \& Stunkard, A. J. (1993). Psychosocial consequences of obesity and dieting: research and clinical findings. In A. J. Stunkard \& T. A. Wadden (Orgs.), Obesity: theory and therapy ( $2^{\underline{a}}$ ed., pp. 163-177). Nova York: Raven.

Yngve, A., Sjöströn, M., Warm, D., Margetts, V., Pérez Rodrigo, C., \& Nissinen, A. (1999). Effective promotion of healthy nutrition and physical activity in Europe requires skilled and competent people; European Master’s Program. Public Health Nutrition, 2, 449-452.

Ana Paula Rodrigues Cavalcanti, mestre em Psicologia Social pela Universidade Federal da Paraíba, é professora no Departamento de Administração da Faculdades de Ensino Superior da Paraíba. Endereço para correspondência: Rua Morise M. Gusmão, 1674/306; João Pessoa, PB; CEP 58071-240. Fone/fax (83) 3231-3047. E-mail: paulacanti@click21.com.br

Mardonio Rique Dias, doutor em Psicologia Social pela Universidade de Brasília, é professor adjunto no Programa de Pós-graduação em Psicologia Social da Universidade Federal da Paraíba e pesquisador do CNPq. Maria José de Carvalho Costa, doutora em Nutrição e Medicina Preventiva pelo Centro de Medicina de Nancy (CNRS/França), é coordenadora do curso de Pós-graduação em Ciências da Nutrição da Universidade Federal da Paraíba. 